\title{
TROPiCS-02: A Phase III study investigating sacituzumab govitecan in the treatment of HR+/HER2- metastatic breast cancer
}

\author{
Hope S Rugo*,1, Aditya Bardia², Sara M Tolaney³, Carlos Arteaga ${ }^{4}$, Javier Cortes ${ }^{5}$, Joohyuk \\ Sohn ${ }^{6}$, Frederik Marmé7, Quan Hong ${ }^{8}$, Rosemary J Delaney ${ }^{8}$, Amir Hafeez ${ }^{8}$, Fabrice André ${ }^{9}$ \\ \& Peter Schmid ${ }^{10}$ \\ ${ }^{1}$ Department of Medicine (Hematology/Oncology), University of California San Francisco Helen Diller Family Comprehensive \\ Cancer Center, San Francisco, CA 94115, USA \\ ${ }^{2}$ Massachusetts General Hospital, Harvard Medical School, Boston, MA 02114, USA \\ ${ }^{3}$ Dana-Farber Cancer Institute, Harvard Medical School, Boston, MA 02215, USA \\ ${ }^{4}$ Harold C. Simmons Comprehensive Cancer Center, University of Texas Southwestern Medical Center, Dallas, TX, USA \\ ${ }^{5}$ Medical Oncology Department, IOB Institute of Oncology, Quirosalud Group, Madrid \& Barcelona, Spain and Senior clinical \\ Researcher, Vall d'Hebron Institute of Oncology (VHIO), Barcelona, Spain \\ ${ }^{6}$ Yonsei Cancer Center, Yonsei University College of Medicine, Seoul, Korea \\ ${ }^{7}$ Medical Faculty Mannheim, Heidelberg University, University Hospital Mannheim, Germany \\ ${ }^{8}$ Immunomedics, Morris Plains, NJ 07950, USA \\ ${ }^{9}$ Department of Medical Oncology, Institut Gustave Roussy, Villejuif, France \\ ${ }^{10}$ Barts Cancer Institute, Queen Mary University of London, London, UK \\ *Author for correspondence: Tel.: +1 415353 7070; Hope.Rugo@ucsf.edu
}

Patients with HR+/HER2- metastatic breast cancer (MBC) whose cancers have progressed despite conventional therapies represent an unmet clinical need. Trop-2, a transmembrane calcium signal transducer, is highly expressed in MBC and plays a role in tumor growth and progression. Sacituzumab govitecan (SG) is a novel antibody-drug conjugate comprising an Trop-2 antibody coupled to SN-38, the active metabolite of irinotecan, via a unique hydrolyzable linker. SG has demonstrated promising activity in a Phase I/II IMMU-132-01 basket study in heavily pretreated solid tumors, including HR+/HER2- MBC. We describe the registrational Phase III TROPiCS-02 study (NCT03901339), evaluating SG versus treatment of physician's choice in HR+/HER2- MBC. Trial registration number: NCT03901339.

First draft submitted: 25 February 2020; Accepted for publication: 9 March 2020; Published online: 30 March 2020

Keywords: antibody-drug conjugate $\bullet$ HER2-negative $\bullet$ HR-positive $\bullet \mathrm{MBC} \bullet$ metastatic breast cancer $\bullet$ sacituzumab govitecan • SN-38 • Trop-2

TROPiCS-02 is an ongoing Phase III, open-label, randomized, multicenter study of sacituzumab govitecan versus treatment of physician's choice (TPC) in patients with HR+/HER2- metastatic breast cancer (MBC) who have received at least two but not more than four prior lines of chemotherapy (NCT03901339). The study assesses both progression-free survival (PFS) and overall response rate (ORR) as the primary end points. Secondary/exploratory end points include overall survival (OS), duration of response (DOR), safety, quality of life (QoL) and blood and tumor biomarkers. The assessments will be conducted by a blinded independent review committee and investigator using Response Evaluation Criteria in Solid Tumors Version 1.1 (RECIST 1.1).

\section{Background \& rationale}

HR+/HER2- breast cancers represent approximately $70 \%$ of all breast cancer subtypes in the USA [1]. Metastatic HR+/HER2- breast cancer remains incurable. Once breast cancer becomes metastatic, only $24 \%$ of patients survive more than 5 years [2]. Patients with HR+/HER2- MBC most commonly have hormone-sensitive disease; thus, endocrine-based therapies, including combinations with targeted agents, such as CDK 4/6 inhibitors, as well as $\mathrm{mTOR}$ and PI3K inhibitors are commonly initial and second- or third-line treatment options [3]. For patients with rapidly progressing or immediately life-threatening visceral metastatic disease, chemotherapy is used earlier in the 


\begin{tabular}{|c|c|c|c|c|c|c|c|}
\hline Agent(s) & Population & $\mathrm{N}$ & Receptor status & ORR (\%) & PFS (months) & OS (months) & Ref. \\
\hline $\begin{array}{l}\text { Metronomic }{ }^{\dagger} \\
\text { Capecitabine } \\
\text { Vinorelbine } \\
\text { Cyclophosphamide }\end{array}$ & $\begin{array}{l}\text { Prior } A, T \text { or } \\
\text { vinorelbine-cyclophosphamide, } \geq 1 \text { prior } \\
\text { chemo/endocrine therapy } \\
\text { for advanced disease }\end{array}$ & 584 & $\begin{array}{l}\mathrm{HR}+(83 \%) \\
\operatorname{TNBC}(16.6 \%)\end{array}$ & $\begin{array}{l}\text { Second-line (22.5) } \\
\text { Third-line (20.8) } \\
\text { Fourth-line (8.8) }\end{array}$ & $\begin{array}{l}\text { Second-line (9.0) } \\
\text { Third-line (5.6) } \\
\text { Fourth-line (4.3) }\end{array}$ & $\begin{array}{l}\text { Second-line (27.2) } \\
\text { Third-line (17.4) } \\
\text { Fourth-line (11.9) }\end{array}$ & [4] \\
\hline Eribulin & $\begin{array}{l}\text { Prior A, T, vinorelbine, } 2 \text { to } \geq 6 \text { prior } \\
\text { chemotherapy for advanced disease } \\
\text { (China) }\end{array}$ & 136 & HR+/HER2- population & 32 & 4.1 & NA & [7] \\
\hline Vinorelbine & $\begin{array}{l}\text { Prior A, T, vinorelbine, } 2 \text { to } \geq 6 \text { prior } \\
\text { chemotherapy for advanced disease } \\
\text { (China) }\end{array}$ & 131 & HR+/HER2- population & 21 & 2.9 & NA & [7] \\
\hline Eribulin & $\begin{array}{l}\text { Prior A, T, 1-2 prior chemotherapy for } \\
\text { advanced disease }\end{array}$ & 265 & $\begin{array}{l}\text { Non-triple negative } \\
\text { sub-population } \\
\text { (HER2-/HR+) }\end{array}$ & NA & NA & 16.4 & [6] \\
\hline Capecitabine & $\begin{array}{l}\text { Prior A, T, 1-2 prior chemotherapy for } \\
\text { advanced disease }\end{array}$ & 286 & $\begin{array}{l}\text { Non-triple negative } \\
\text { sub-population } \\
(\mathrm{HER} 2-/ \mathrm{HR}+\text { ) }\end{array}$ & NA & NA & 16.1 & [6] \\
\hline Eribulin & $\begin{array}{l}\text { Prior } A \text { and } T, \geq 2 \text { prior chemotherapy } \\
\text { (median } 4 \text { ) }\end{array}$ & 508 & $\begin{array}{l}\text { HR }+(64 \%) \\
\text { HER2- }(73 \%) \\
\text { TNBC }(18 \%)\end{array}$ & 12 & 3.7 & 13.1 & [5] \\
\hline
\end{tabular}

course of therapy [3]. Once patients have developed progressive disease on multiple sequential endocrine therapies, or have primary endocrine resistance, subsequent lines of therapy often consist of single-agent chemotherapy [3]. Response rates to later-line therapies are typically low due to intrinsic or acquired treatment resistance (Table 1) [47]. A median PFS of approximately 4-5 months has been reported for patients with HR+/HER2- MBC with later-line chemotherapies [4,5,7]. Progressive disease on later lines of chemotherapy in addition to endocrine therapy significantly limits treatment options for patients with HR+/HER2- MBC; representing a substantial unmet need for safe, effective treatment options for these patients.

\section{ADC for the treatment of cancers}

Antibody-drug conjugates (ADCs) represent an evolving class of therapeutic agents specifically designed to improve on the delivery of chemotherapeutic agents by exploiting the target-selectivity of monoclonal antibodies [8]. ADCs are composed of a monoclonal antibody and potent cytotoxic chemotherapy (payload) conjugated via a linker moiety. The monoclonal antibody targets antigens selected for their higher expression on tumor cells compared with normal cells; the payload is then internalized and released when the chemical linker is broken by cellular conditions, such as low $\mathrm{pH}$ or proteasome-mediated degradation. By targeting antigen- or receptor-presenting tumor cells, this limits off-target toxicity and therefore should reduce the level of toxicity normally associated with chemotherapy, a treatment that does not discriminate between normal and malignant cells [8].

The antibody portion of the ADC that binds to the tumor cell surface can be individualized for specific cell surface antigens or receptors [8], thereby making it possible to adapt ADCs to treat a wide range of cancers. Several ADCs have been approved by the US FDA for the treatment of hematological malignancies and solid tumors, with approximately 80 ADCs that are being studied in clinical trials [8]. Two ADCs are currently approved for use in HER2+ breast cancer [9,10]. The success of ADCs to date clearly demonstrates the clinical potential of these agents, with more ADCs being developed to further enhance the breast cancer treatment landscape [11]. ADC strategies may be an attractive therapeutic option for patients with HR+/HER2- MBC, especially patients receiving later-line therapy who need novel and more effective treatment. Though HR+/HER2- breast cancers lack the HER 2 oncogenic driver of tumor progression, preclinical models of the disease suggest that other receptors that may play a key role in tumorigenesis of this disease and are overexpressed in tumors and thus may be potential targets for the design of ADC strategies.

\section{Targeting Trop-2 as a breast cancer treatment strategy}

Trop-2 is a calcium signal transducer discovered to play a role in anchorage-independent cell growth, migration and invasion [12]. Trop-2 has been implicated in several intracellular signaling pathways, such as MAPK, Raf and 


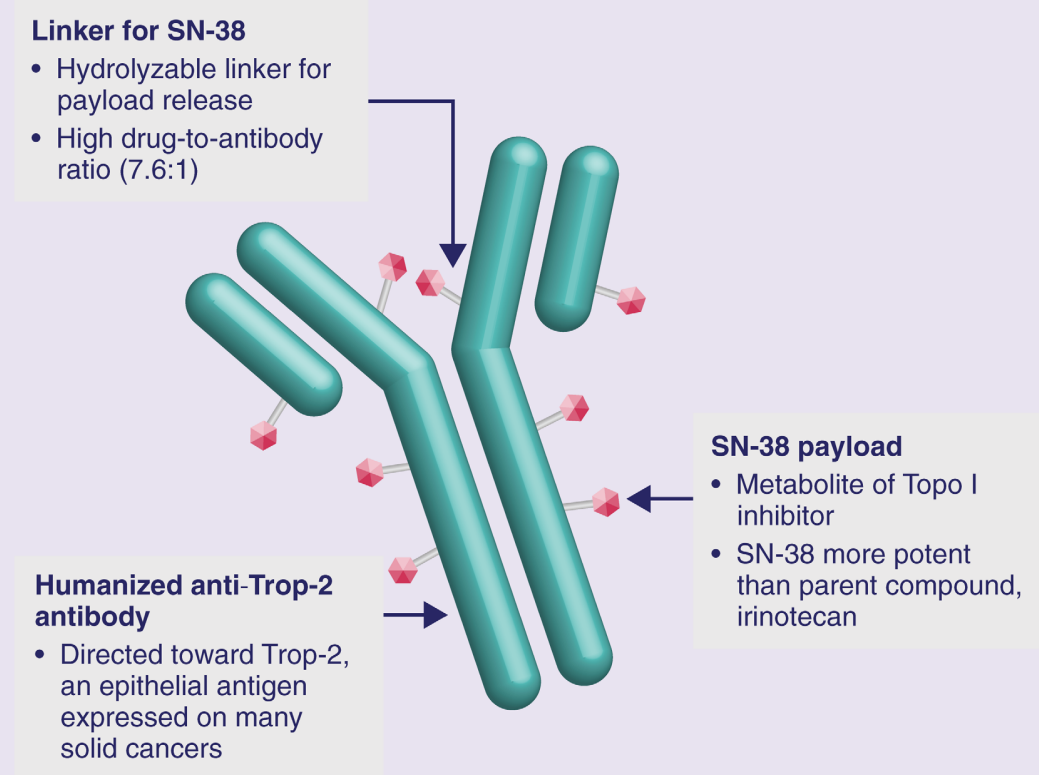

Figure 1. Sacituzumab govitecan is a trophoblast cell-surface antigen 2-directed antibody drug conjugate.

Sacituzumab govitecan is a novel antibody-drug conjugate composed of an anti-Trop-2 antibody coupled to SN-38, the active metabolite of irinotecan, via a unique hydrolyzable linker. Sacituzumab govitecan is an investigational anti-Trop-2 ADC that has not yet been approved by any regulatory agency for the treatment of breast cancer. ADC: Antibody-drug conjugate.

NF-KB, further contributing to tumor cell survival and progression [12,13]. Overexpressing Trop-2 has been found to be necessary and sufficient to stimulate tumor growth [13].

Trop-2 is highly overexpressed in most human solid epithelial cancers, with lower expression reported in certain normal tissues [13,14]. In an immunohistochemistry analysis of 702 consecutive breast cancer samples, Trop-2 expression was detected with a wide range of breast cancer subtypes [15]. HR+/HER2- breast cancer has shown significantly higher Trop-2 expression than have other breast cancer subtypes, including HER2+ [14]. Molecular markers that significantly influence the biological progress of tumors can also provide important prognostic information. Overexpression of Trop- 2 has been associated with more aggressive breast cancers $[7,14]$ and unfavorable prognosis with regard to patient survival outcomes (e.g., OS, disease-free survival) $[15,16]$. Taken together, Trop-2 is a potentially valuable novel therapeutic target and prognostic biomarker for patients with advanced HR+/HER2MBC.

\section{Sacituzumab govitecan: a Trop-2 directed ADC}

Sacituzumab govitecan (SG) is an ADC that utilizes the humanized RS7 (hRS7) anti-Trop-2 monoclonal antibody and a proprietary hydrolyzable linker to selectively deliver $\mathrm{SN}-38$, the active metabolite of irinotecan [17] to Trop-2-expressing tumor cells (Figure 1) [18]. SG has a higher than most drug-to-antibody ratio compared with other ADCs [13]. This ADC permits a high site-specific coupling of 7.6 molecules of SN-38 per monoclonal antibody without altering pharmacokinetics or reducing the therapeutic index of the conjugated antibody. This delivery advantage ensures high concentrations of SN-38. Once SG is administered, the anti-Trop-2 monoclonal antibody binds to Trop-2 expressed on the tumor cell surface (Figure 2). The Trop-2-bound hRS7 complex is then internalized and trafficked intracellularly to lysosomes. Free SN-38 is released from SG through antibody catabolism followed by hydrolysis of the linker within the lysosome. SN-38, a moderately toxic chemotherapeutic agent with activity in the low nanomolar range, then targets Topo I, resulting in DNA damage and cellular apoptosis. Given its membrane-permeable nature, free SN-38 may then leave the cell and elicit antitumor effects in adjacent tumor cells. SG will also release SN-38 in the tumor microenvironment pre-internalization through hydrolysis of the linker, 


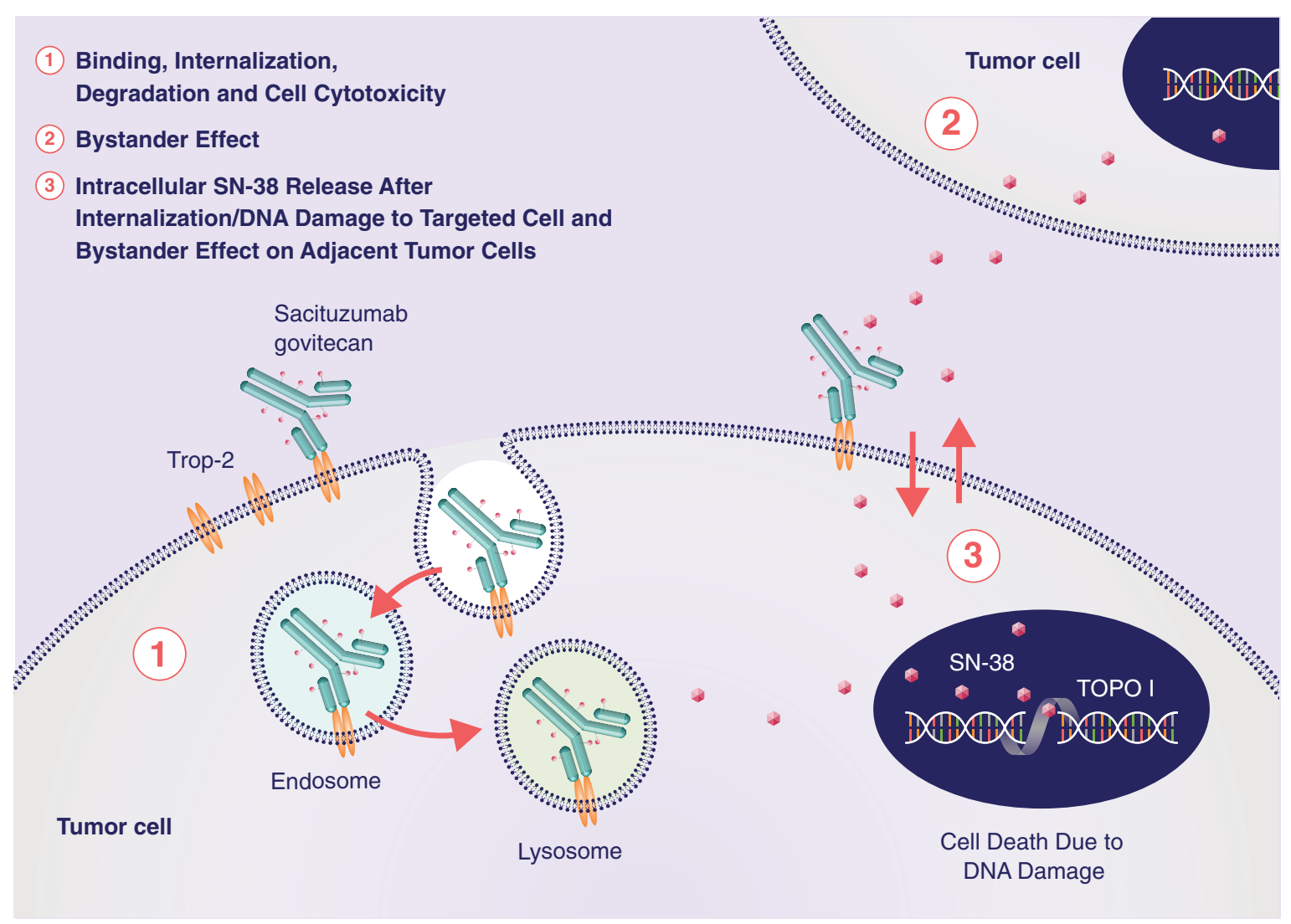

Figure 2. The mechanism of action of sacituzumab govitecan. The specialized design of sacituzumab govitecan delivers high concentrations of cytotoxic SN-38 to Trop-2-expressing tumor cells as well as adjacent tumor cells in the tumor microenvironment.

allowing for a concentration of SN-38 to adjacent tumor cells [13], leading to a bystander effect. Extracellularly released SN-38 may also act on the specific tumor cell to which the ADC is bound. Thus, SG is an ADC that appears to potentially be an effective therapeutic strategy for the treatment of cancer, including tumors with antigen heterogeneity.

The safety, pharmacokinetics and clinical activity of SG monotherapy have been investigated in relapsed/refractory, advanced solid tumors unselected for Trop-2 expression, including triple-negative breast cancer (TNBC) and non-small-cell lung, urinary bladder, ovarian and HR+ breast cancers in a Phase I/II, open-label, single-arm, multicenter, basket study (IMMU-132-01; NCT01631552) [19]. In this basket study, SG had a moderate and manageable safety profile and was tolerable at doses of up to $12 \mathrm{mg} / \mathrm{kg}$ on days 1 and 8 of a 21-day cycle, with neutropenia as the only dose-limiting toxicity [19]. The $10 \mathrm{mg} / \mathrm{kg}$ dose exhibited a tolerable safety profile when administered in repeated cycles and thus was selected for clinical development.

In the IMMU-132-01 basket study, SG demonstrated durable objective responses in the cohort of 108 heavily pretreated patients with TNBC [20]. Patients were treated with $10 \mathrm{mg} / \mathrm{kg}$ on days 1 and 8 of each 21-day cycle until progressive disease or unacceptable toxicity. At the time of data cutoff (1 December 2017; median follow-up, 9.7 months) ORR was 33\% (36/108; three complete responses [CRs] and 33 partial responses [PRs]), with a median DOR of 7.7 months (95\% CI: 4.9-10.8) by local assessment. Median PFS was 5.5 months (95\% CI: 4.1-6.3) and median OS was 13.0 months (95\% CI: 11.2-13.7). Very few patients (3\%) discontinued treatment due to adverse events (AEs). Myelosuppression (grade 3/4 neutropenia and anemia, $42 \%$ and $21 \%$, respectively) and diarrhea (mostly grade 1 ; grade $3 / 4,8 \%$ ) were the primary AEs but were manageable with routine supportive care (e.g., use of growth factors). No cases of severe treatment-related neuropathy or cardiac AEs or grade $3 / 4$ peripheral neuropathy were observed. Four deaths were reported though none were considered treatment related. This safety profile was also consistent with the overall safety population of the basket study. Because of the clinical benefit observed in this cohort, SG received breakthrough therapy designation by the US FDA for the treatment 


\section{TROPiCS -02}

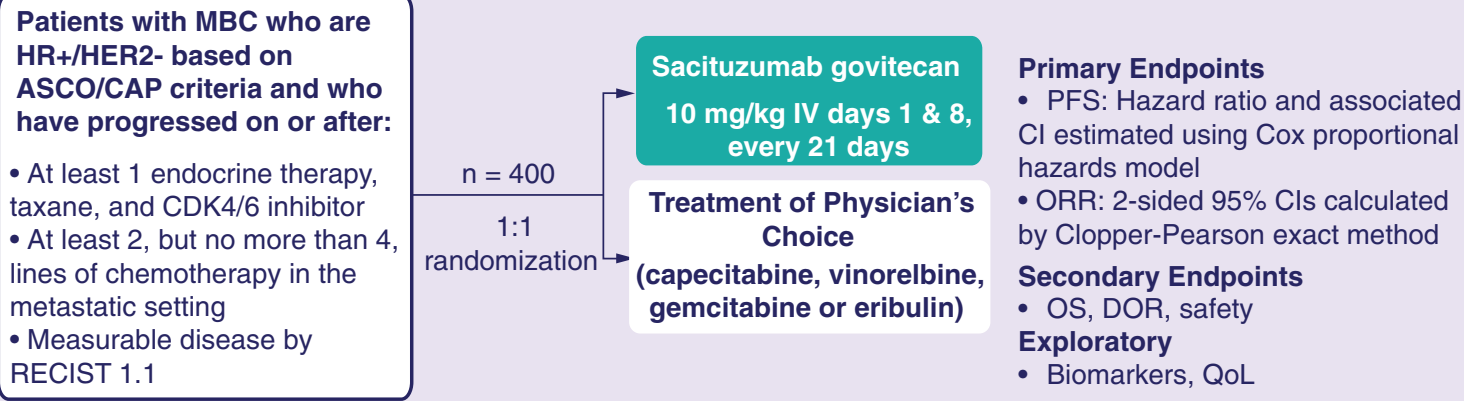

- At least 1 endocrine therapy, taxane, and CDK4/6 inhibitor - At least 2, but no more than 4 , lines of chemotherapy in the metastatic setting

- Measurable disease by RECIST 1.1

Sacituzumab govitecan Primary Endpoints

$10 \mathrm{mg} / \mathrm{kg}$ IV days 1 \& 8, . PFS: Hazard ratio and associated every 21 days $\quad \mathrm{Cl}$ estimated using Cox proportiona hazards model

- ORR: 2-sided $95 \%$ Cls calculated by Clopper-Pearson exact method

Secondary Endpoints

- OS, DOR, safety

Exploratory

- Biomarkers, QoL

Stratification

- Visceral metastases $(\mathrm{Y} / \mathrm{N})$

- Endocrine therapy in metastatic setting $>6$ months $(\mathrm{Y} / \mathrm{N})$

- 2 vs $3 / 4$ prior lines of chemotherapies

Figure 3. Study design.

ASCO/CAP: American Society of Clinical Oncology/College of American Pathologists criteria; DOR: Duration of response; HR: Hormone-receptor; IV: Intravenous; MBC: Metastatic breast cancer; ORR: Overall response rate; OS: Overall survival; PFS: Progression-free survival; QoL: Quality of life; RECIST 1.1: Response Evaluation Criteria in Solid Tumors version 1.1.

of patients with TNBC with $\geq 2$ prior therapies for metastatic disease. The confirmatory multicenter, randomized Phase III ASCENT trial (NCT02574455) is underway in patients with metastatic TNBC who have failed at least two prior lines of chemotherapy.

SG also demonstrated significant single-agent activity in the cohort of 54 patients enrolled between February 2015 and June 2017 who had HR+/HER2- MBC [21]. Patients (median age, 54 years) had received a median of three (range, 1-6) prior hormonal therapies and two (range, 0-9) chemotherapies in the metastatic setting. The primary sites of metastatic disease for the majority of patients at study entry were the liver (82\%) and bone (80\%). As of data cutoff (30 April 2018), SG monotherapy demonstrated significant clinical activity, with a confirmed ORR of 31\% (17/54; all PRs) in the treated population and a clinical benefit rate (including CR, PR and stable disease $\geq 6$ months) of $48 \%$ (26/54) in patients based on local assessment using RECIST 1.1 [21]. Responses were durable, with an estimated median DOR of 7.4 months (95\% CI: 4.4-18.3) and PFS of 6.8 months (95\% CI: 4.6-8.9). SG showed a manageable safety profile, with a low incidence of grade $3 / 4$ febrile neutropenia (4\%) and diarrhea (2\%) among the 50 patients with available data. SG was also well tolerated, with $3.7 \%$ of patients discontinuing treatment due to AEs (grade 3 neutropenia not recovered within 3 weeks; grade 3 diarrhea/dehydration). AEs were managed with supportive medication or dose modifications. There were no cases of interstitial lung disease, rash, severe treatment-related neuropathy or cardiac AEs and no treatment-related deaths were reported. These data demonstrate the efficacy and safety of SG monotherapy and provide rationale for additional larger studies assessing SG for the treatment of HR+/HER2- MBC.

\section{TROPICS-02}

\section{Study design}

TROPiCS-02 is an open-label, randomized, multicenter Phase III study that will evaluate the efficacy and safety of SG versus TPC in patients with metastatic or locally advanced inoperable HR+/HER2- MBC, after failure of at least two but no more than four prior chemotherapy regimens for metastatic disease (Figure 3) [22]. The primary endpoints are PFS and ORR determined by local investigator review using RECIST 1.1. Key secondary endpoints include OS, clinical benefit rate, DOR, QoL between treatment arms using European Organization for the Research and Treatment of Cancer QoL questionnaire version 30 (QLQ-C30) and the European Quality of Life (EuroQOL) EQ-5D-5L instruments, treatment-related symptoms using a set of nine relevant symptom concepts from the 
Patient-Reported Outcomes version of the Common Terminology Criteria for Adverse Events (PRO-CTCAE) and safety and tolerability. The study opened for accrual April 2019 and is currently recruiting patients. Approximately 400 patients will be randomized in this study and will be recruited from 129 sites across the USA, Canada, the UK, France, Spain, Italy, Germany, Belgium and the Netherlands.

\section{Eligibility criteria}

Patients must be aged $\geq 18$ years with histologically confirmed HR+/HER2- MBC according to American Society of Clinical Oncology/College of American Pathologists criteria and have been previously treated with at least two but no more than four prior systemic chemotherapy regimens for $\mathrm{MBC}$, including at least one prior taxane in any setting, at least one prior anticancer hormonal treatment and at least one CDK4/6 inhibitor in any setting. Disease progression on the most recent therapy documented by computed tomography (CT) or MRI and at least one measurable target lesion according to RECIST 1.1 is required (patients with bone disease only are not eligible). Patients must also be eligible per investigator for at least one of four prespecified TPC agents (capecitabine, eribulin, vinorelbine or gemcitabine). Eligibility also includes an Eastern Cooperative Oncology Group Performance Status of $\leq 1$ and adequate bone marrow (hemoglobin $>9 \mathrm{~g} / \mathrm{dl}$, absolute neutrophil count $>1500 / \mathrm{mm}^{3}$ and platelets $>100,000 / \mathrm{mm}^{3}$ ), renal (calculated creatinine clearance $\geq 30 \mathrm{ml} / \mathrm{min}$ ) and hepatic (bilirubin $\leq 1.5 \mathrm{x}$ upper limit of normal [ULN], AST/ALT $\leq 2.5 \times$ ULN [or $\leq 5 \times$ ULN if known liver metastasis] and ALP $\leq 2.5 \times$ ULN) function.

Patients will be excluded if they have received prior Topo 1 inhibitors, have a history of significant cardiovascular disease or a clinically significant ECG abnormality, have active CNS metastases unless stable for at least 4 weeks, have an active infection requiring intravenous systemic therapy or active chronic inflammatory bowel disease with previous bowel obstruction or have additional concurrent medical or psychiatric conditions that may confound study interpretation or prevent completion of study procedures and assessments.

\section{Study procedures}

Patients will be randomized $1: 1$ to receive $10 \mathrm{mg} / \mathrm{kg}$ of SG administered intravenously on days 1 and 8 for every 21day cycle or single-agent standard of care TPC (eribulin, capecitabine, gemcitabine or vinorelbine). Randomization will be stratified by the presence (versus absence) of visceral metastases, duration of endocrine treatment in the metastatic setting ( $\geq 6$ months vs not) and the number of prior lines of chemotherapy (two vs three or four). Patients are treated until disease progression, unacceptable toxicity, withdrawal of consent or per investigator judgment. Treatment is allowed beyond progression if judged clinically beneficial by investigators.

\section{Outcome measures}

Tumor assessments will be performed using RECIST 1.1. Response assessments include CT/MRI scans (chest, abdomen, pelvis and other areas of involvement) every 6 weeks for 54 weeks and then subsequently every 9 weeks. A bone scan will be performed within 6 weeks prior to first dose and within a target of 1-2 weeks following a CR. Safety is assessed throughout and is graded based on National Cancer Institute CTCAE v5.0. QoL will be assessed at baseline and on day 1 of each subsequent cycle. Trop- 2 expression, tumor mutations and the expression of other key biomarkers used to identify potential correlation with clinical outcomes-related end points will be evaluated in blood samples (collected at baseline, predose on day 1 of cycle 2 and at the end of treatment) and tumor tissue (archived tumor tissue or most recent formalin-fixed paraffin-embedded tissues).

\section{Statistical analysis methods}

PFS is defined as the time from randomization to the first documented disease progression or death (whichever occurs first), according to local investigator review. ORR is defined as the proportion of patients who have a confirmed best overall response of CR or PR, according to blind independent review committee using RECIST 1.1. OS is defined as the time from randomization to death from any cause. DOR is defined as the time from a documented response until the first documented disease progression or death (whichever occurs first).

Appropriate censoring rules will be applied for determining PFS and OS by using the Kaplan-Meier method for survival estimates. The stratified log-rank test will be used to assess between-group differences and the stratified Cox proportional hazards model will be fitted to compute hazard ratios and corresponding $95 \%$ CIs. The stratification factors used at randomization were applied to all stratified analyses. 


\section{Conclusion}

ADCs targeting Trop-2 may be a safe and effective novel approach to the treatment of patients with HR+/HER2MBC refractory to endocrine therapy and who have relatively short survival outcomes on standard chemotherapy. SG is a Trop-2-directed ADC that has the potential to make a great impact on the treatment landscape for TNBC and has shown encouraging efficacy with manageable safety in patients with HR+/HER2- MBC. The unique properties of SG include its higher drug:antibody ratio (7.6:1), a less toxic payload and the additional effect of delivering concentrations of the payload to low Trop-2-expressing cells in the tumor microenvironment (bystander effect). Clinical data have also shown a manageable safety profile with the observed adverse events of neutropenia and diarrhea, but no cases of severe neuropathy, interstitial lung disease or rash have been observed. Even in a heavily pretreated population, no treatment-related deaths have occurred. It is hoped that this tolerable safety profile of SG will allow for ease in future use in combinations with traditional chemotherapies or immunotherapies.

The TROPiCS-02 Phase III, randomized, multicenter, registrational study described in this article will determine whether SG monotherapy can improve PFS and ORR in patients with HR+/HER2- MBC previously treated with $\geq 1$ prior endocrine therapy and two to four lines of chemotherapy compared with standard chemotherapy TPC. Positive results from TROPiCS-02 will lead to the availability of a novel, effective treatment option other than chemotherapy for patients with HR+/HER2- MBC and address an important unmet medical need in the field of breast oncology.

\section{Executive summary}

\section{Background}

- Patients with HR+/HER2- metastatic breast cancer (MBC) who have exhausted conventional therapies have a poor prognosis (median progression-free survival [PFS] of 4-5 months for later-line chemotherapy) and limited treatment options.

- Breast cancers overexpress Trop-2, a transmembrane calcium signal transducer with a role in tumor growth and progression, providing a rationale for anti-Trop-2 treatment strategies for patients with MBC.

Sacituzumab Govitecan

- Sacituzumab govitecan (SG) is a novel antibody-drug conjugate comprising an anti-Trop-2 monoclonal antibody conjugated to $\mathrm{SN}-38$ (active metabolite of irinotecan) in a high drug-to-antibody ratio of 7.6. It has a unique hydrolyzable linker that allows release of SN-38 intracellularly and in the tumor microenvironment.

- In the IMMU-132-01 study, cohort of patients with HR+/HER2- MBC who had $\geq 1$ prior endocrine therapy, treatment with SG resulted in a confirmed overall response rate of $31 \%$ with a clinical benefit rate of $48 \%$; median PFS was 6.8 months.

TROPiCS-02 Phase III study

- TROPiCS-02 evaluates SG versus treatment of physician's choice in patients with HR+/HER2- MBC who received at least two prior lines of chemotherapy.

- Patients must be aged $\geq 18$ years, have histologically confirmed HR+/HER2- MBC, relapsed/refractory to two to four prior systemic chemotherapy regimens for $\mathrm{MBC}$, including at least one prior anticancer hormonal treatment and at least one CDK4/6 inhibitor in any setting.

- Approximately 400 patients will be randomized $1: 1$ to receive SG $10 \mathrm{mg} / \mathrm{kg}$ intravenously on days 1 and 8 (21-day cycle) or treatment of physician's choice (eribulin, capecitabine, gemcitabine or vinorelbine) administered as specified per label.

- Randomization will be stratified by the presence/absence of visceral metastases, whether or not endocrine treatment was given in the metastatic setting for 6 months or more and the number of prior lines (two vs three or four).

- Primary end points are PFS and overall response rate.

- Secondary end points include overall survival, duration of response, quality of life and safety.

Conclusion

- Results from the randomized registrational Phase III TROPiCS-02 study will provide robust efficacy and safety data for SG compared with single-agent chemotherapy that may lead to a novel, effective later-line treatment option for patients with HR+/HER2- MBC to address a dire unmet medical need.

\section{Infographic}

An infographic accompanies this paper at the end ofthe references section. To download the infographic that accompanies this paper, please visit the journal website at: www.futuremedicine.com/doi/10.2217/fon-2020-0163 
Author contributions

All the authors were involved in the design and/or conduct of the study. All authors have contributed to the preparation and writing of the manuscript and approved the final manuscript.

\begin{abstract}
Acknowledgments
We thank the patients and their caregivers for helping us realize the possibilities of this research as well the dedicated clinical trial investigators and their devoted team members participating in the trial.
\end{abstract}

Financial \& competing interests disclosure

The study was sponsored by Immunomedics, Inc. and was designed through a collaboration between the sponsor and the lead investigators. HS Rugo has received research grants to the UC Regents from Pfizer, Novartis, Eli Lilly, F. Hoffmann-La Roche, Macrogenics, Merck, OBI Pharma, Eisai, Daiichi Sankyo, Odonate, Seattle Genetics and Immunomedics, Inc.; and has received travel, accommodations or expenses from Puma Biotechnology, Mylan Pharmaceutics, Lilly, Pfizer, Amgen, F. Hoffmann-La Roche and AstraZeneca. A Bardia has served as a consultant/advisor for Biotheranostics, Pfizer, Novartis, Genentech, Merck, Radius Health, Immunomedics, Spectrum Pharmaceuticals, Taiho Pharmaceutical, Sanofi, Daiichi Pharma/Astra Zeneca, Puma Biotechnology, Lilly and Philipps; has received research funding from Genentech/F Hoffman-La Roche, Novartis, Pfizer, Merck, Sanofi, Radius Health, Immunomedics, Mersana Therapeutics, Innocrin Pharmaceuticals and Biothernostics. SM Tolaney has served as a consultant/advisor for Novartis, Eli Lilly, Pfizer, Immunomedics, Merck, NanoString Technologies, Nektar Therapeutics, Eisai, AstraZeneca, Genentech/F. Hoffmann-La Roche, Puma Biotechnology, Sanofi, Athenex, Seattle Genetics, Silverback Therapeutics, G1 Therapeutics, Paxman, Odonate, AbbVie, Bristol-Myers Squibb and Celldex Therapeutics; and has institutional research funding from AstraZeneca, Eli Lilly, Merck, Nektar Therapeutics, Novartis, Pfizer, Genentech/F. Hoffmann-La Roche, Eisai, NanoString Technologies, Immunomedics, AstraZeneca, Eli Lilly, Merck, Nektar Therapeutics, Novartis, Pfizer, Eisai, NanoString Technologies, Exelixis, Cyclacel, Odonate and Bristol-Myers Squibb. C Arteaga has served as a consultant/advisor for AbbVie, Novartis, Eli Lilly, Sanofi, Radius Health, Taiho Pharmaceutical, Puma Biotechnology, Merck, H3 Biomedicine OrigiMed, Immunomedics, Petra Pharma, G1 Therapeutics, Athenex, Daiichi Sankyo and Clovis; has ownership interest (stock, stock options or other ownership interest excluding diversified mutual funds) in Provista and Y-TRAP; and has received research funding from Pfizer, Eli Lilly, Bayer HealthCare Pharmaceuticals and Takeda; is on the Scientific Advisory Board for Susan G Komen for the Cure Breast Cancer Foundation and is a Scientific Steering Committee member for TROPiCS-02, the subject of the manuscript. J Cortes has a patent with MedSIR; has served as a consultant/advisor for Genentech/F Hoffmann-La Roche, Celgene, Cellestia, AstraZeneca, Biothera Pharmaceutical, Merus, Seattle Genetics, Daiichi Sankyo, Erytech, Athenex, Polyphor, Lilly, Servier, Merck Sharp \& Dohme, GSK, Leuko, Bioasis, Clovis Oncology; has received honoraria from Genentech/F Hoffmann-La Roche, Novartis, Celgene, Eisai, Pfizer, Samsung Bioepis, Lilly, Merck Sharp \& Dohme and Daiichi Sankyo; has research funding from F Hoffmann-La Roche, ARIAD Pharmaceuticals, AstraZeneca, Baxalta GMBH/Servier Affaires, Bayer HealthCare Pharmaceuticals, Eisai, Guardant Health, Merck Sharp \& Dohme, Pfizer, PIQUR Therapeutics, Puma Biotechnology and Queen Mary University of London; and has received travel, accommodations or expenses from Genentech/F Hoffmann-La Roche, Novartis, Eisai, Pfizer and Daiichi Sankyo. J Sohn has received research funding from Bayer HealthCare Pharmaceuticals, GlaxoSmithKline, Contessa, Daiichi Sankyo, F Hoffmann-La Roche, Novartis, AstraZeneca, Eli Lilly, Merck, Sharp \& Dohme and Pfizer. F Marmé has served as a consultant/advisor for AstraZeneca, Tesaro, F Hoffmann-La Roche, Novartis, Amgen, Pfizer, CureVac, PharmaMar, Eisai, Bristol-Myers Squibb, Genomic Health; and has provided contracted research for and received honoraria from Immunomedics. Q Hong is an employee of and holds ownership interest in Immunomedics; has a spouse/partner who is an employee of and holds ownership interest in Merck; and has received travel, accommodations or expenses from Advaxis. RJ Delaney and A Hafeez are employees of and hold ownership interest in Immunomedics. F André has received research funding from AstraZeneca, Pfizer, Eli Lilly, F Hoffmann-La Roche, Novartis and Daiichi Sankyo. P Schmid has a spouse/partner who is a consultant of Genentech/F Hoffmann-La Roche; has received research funding from Genentech/F Hoffmann-La Roche, OncoGenex Pharmaceuticals and Novartis; has received honoraria from Bayer HealthCare Pharmaceuticals, Eisai, Bristol-Myers Squibb, Puma Biotechnology, Pfizer, AstraZeneca, Novartis, F Hoffmann-La Roche, Merck, Boehringer Ingelheim; and was an uncompensated steering committee member for the IMpassion130 trial. The authors have no other relevant affiliations or financial involvement with any organization or entity with a financial interest in or financial conflict with the subject matter or materials discussed in the manuscript apart from those disclosed.

Medical writing assistance was provided by Shala Thomas, PhD of Team 9 Science and was funded by Immunomedics, Inc. 


\section{Ethical conduct of research}

The independent review/ethics committee at each site approved the protocol. All patients provided written informed consent. The study was conducted according to the principles of the Declaration of Helsinki and the International Conference on Harmonisation Guidelines for Good Clinical Practice.

\section{Open access}

This work is licensed under the Attribution-NonCommercial-NoDerivatives 4.0 Unported License. To view a copy of this license, visit http://creativecommons.org/licenses/by-nc-nd/4.0/

\section{References}

Papers of special note have been highlighted as: $\bullet$ of interest; $\bullet \bullet$ of considerable interest

1. Howlader N, Altekruse SF, Li CI et al. US incidence of breast cancer subtypes defined by joint hormone receptor and HER2 status. J. Natl Cancer Inst. 106(5), 1-8 (2014).

- Provides the incidence of breast cancer subtypes in the USA, including HR+/HER2- subtypes, illustrating the dire need for novel agents.

2. Harb WA. Management of patients with hormone receptor-positive breast cancer with visceral disease: challenges and treatment options. Cancer Manag. Res. 7, 37-46 (2015).

3. Matutino A,Joy AA, Brezden-Masley C, ,et al.,,Hormone receptor-positive, HER2-negative metastatic breast cancer: redrawing the lines. Curr. Oncol. 25(Suppl.1), S131-S141 (2018).

4. Cazzaniga ME, Pinotti G, Montagna E et al. Metronomic chemotherapy for advanced breast cancer patients in the real world practice: final results of the VICTOR-6 study. Breast 48, 7-16 (2019).

5. Cortes J, O'Shaughnessy J, Loesch D et al. Eribulin monotherapy versus treatment of physician's choice in patients with metastatic breast cancer (EMBRACE): a Phase III open-label randomised study. Lancet 377(9769), 914-923 (2011).

6. Twelves C, Awada A, Cortes J et al. Subgroup analyses from a Phase III, open-label, randomized study of eribulin mesylate versus capecitabine in pretreated patients with advanced or metastatic breast cancer. Breast Cancer (Auckl.) 10, 77-84 (2016).

7. Yuan $\mathrm{P}, \mathrm{Hu} X$, Sun $\mathrm{T}$ et al. Eribulin mesilate versus vinorelbine in women with locally recurrent or metastatic breast cancer: a randomised clinical trial. Eur. J. Cancer 112, 57-65 (2019).

8. Coats S, Williams M, Kebble B et al. Antibody-drug conjugates: future directions in clinical and translational strategies to improve the therapeutic index. Clin. Cancer Res. 25(18), 5441-5448 (2019).

9. Dieras V, Miles D, Verma $S$ et al. Trastuzumab emtansine versus capecitabine plus lapatinib in patients with previously treated HER2-positive advanced breast cancer (EMILIA): a descriptive analysis of final overall survival results from a randomised, open-label, Phase III trial. Lancet Oncol. 18(6), 732-742 (2017).

10. von Minckwitz G, Huang CS, Mano MS et al. Trastuzumab emtansine for residual invasive HER2-positive breast cancer. N. Engl. J. Med. 380(7), 617-628 (2019).

11. Trail PA, Dubowchik GM, Lowinger TB. Antibody drug conjugates for treatment of breast cancer: novel targets and diverse approaches in ADC design. Pharmacol. Ther. 181, 126-142 (2018).

12. Shvartsur A, Bonavida B. Trop2 and its overexpression in cancers: regulation and clinical/therapeutic implications. Genes Cancer 6(3-4), 84-105 (2015).

13. Goldenberg DM, Stein R, Sharkey RM. The emergence of trophoblast cell-surface antigen 2 (TROP-2) as a novel cancer target. Oncotarget 9(48), 28989-29006 (2018).

- Provides an overview of the rationale for Trop-2 as an emerging key biomarker and target for certain cancers, including HR+/HER- breast cancers, including discussion around the potential of strategies targeting Trop-2.

14. Vidula N, Yau C, Rugo HS. Trop2 gene expression (Trop2e) in primary breast cancer (BC): correlations with clinical and tumor characteristics. J. Clin. Oncol. 35(15 Suppl.), 1075 (2017).

- Reports that Trop-2 expression in HR+/HER2- breast cancers is significantly higher than other breast cancer subtypes, including HER2+.

15. Ambrogi F, Fornili M, Boracchi P et al. Trop-2 is a determinant of breast cancer survival. PLoS ONE 9(5), e96993 (2014).

- Demonstrates that membrane-associated Trop-2 has an unfavorable prognostic impact on survival for patients with breast cancer.

16. Lin H, Huang JF, Qiu JR et al. Significantly upregulated TACSTD2 and cyclin D1 correlate with poor prognosis of invasive ductal breast cancer. Exp. Mol. Pathol. 94(1), 73-78 (2013).

17. Mathijssen RH, van Alphen RJ, Verweij J et al. Clinical pharmacokinetics and metabolism of irinotecan (CPT-11). Clin. Cancer Res. 7(8), 2182-2194 (2001).

18. Goldenberg DM, Cardillo TM, Govindan SV, Rossi EA, Sharkey RM. Trop-2 is a novel target for solid cancer therapy with sacituzumab govitecan (IMMU-132), an antibody-drug conjugate (ADC). Oncotarget 6(26), 22496-22512 (2015). 
19. Starodub AN, Ocean AJ, Shah MA et al. First-in-human trial of a novel anti-Trop- 2 antibody-SN-38 conjugate, sacituzumab govitecan, for the treatment of diverse metastatic solid tumors. Clin. Cancer Res. 21(17), 3870-3878 (2015).

-. Reports the first-in-human study of sacituzumab govitecan in a variety of relapsed/refractory metastatic solid tumors; sacituzumab govitecan had therapeutic activity with an acceptable safety profile in patients with difficult-to-treat cancers.

20. Bardia A, Mayer IA, Vahdat LT et al. Sacituzumab govitecan-hziy in refractory metastatic triple-negative breast cancer. N. Engl. J. Med. 380(8), 741-751 (2019).

-• Reports that sacitumumab govitecan demonostrated a durable overall response rate of $33 \%$ by local assessment with a manageable safety profile in a population of heavily pretreated patients with triple negative breast cancer. Based on these data, sacituzumab govitecan received breakthrough designation from the US FDA in patients with triple negative breast cancer who have failed prior therapies for metastatic disease.

21. Bardia A, Diamond JR, Vahdat L et al. Efficacy of sacituzumab govitecan (anti-Trop-2-SN-38 antibody-drug conjugate) for treatment-refractory hormone-receptor positive (HR+)/HER2 negative metastatic breast cancer (mBC). Presented at: 2018 American Society of Clinical Oncology (ASCO) Annual Meeting. Chicago, IL, USA, (2018).

-• Reports that sacituzumab govitecan showed single-agent clinical activity in heavily pretreated HR+/HER2- metastatic breast cancer patients, with an overall response rate of $31 \%$, a clinical benefit rate of $48 \%$ and a median progression-free survival of 6.8months.

22. Rugo HS, Bardia A, Tolaney SM et al. TROPiCS-02: Phase III study of sacituzumab govitecan (SG) in relapsed/refractory hormonal receptor-positive (HR+)/human epidermal growth factor receptor 2 negative (HER2-) metastatic breast cancer (MBC). Presented at: San Antonio Breast Cancer Symposium. San Antonio, TX, USA, (2019). 

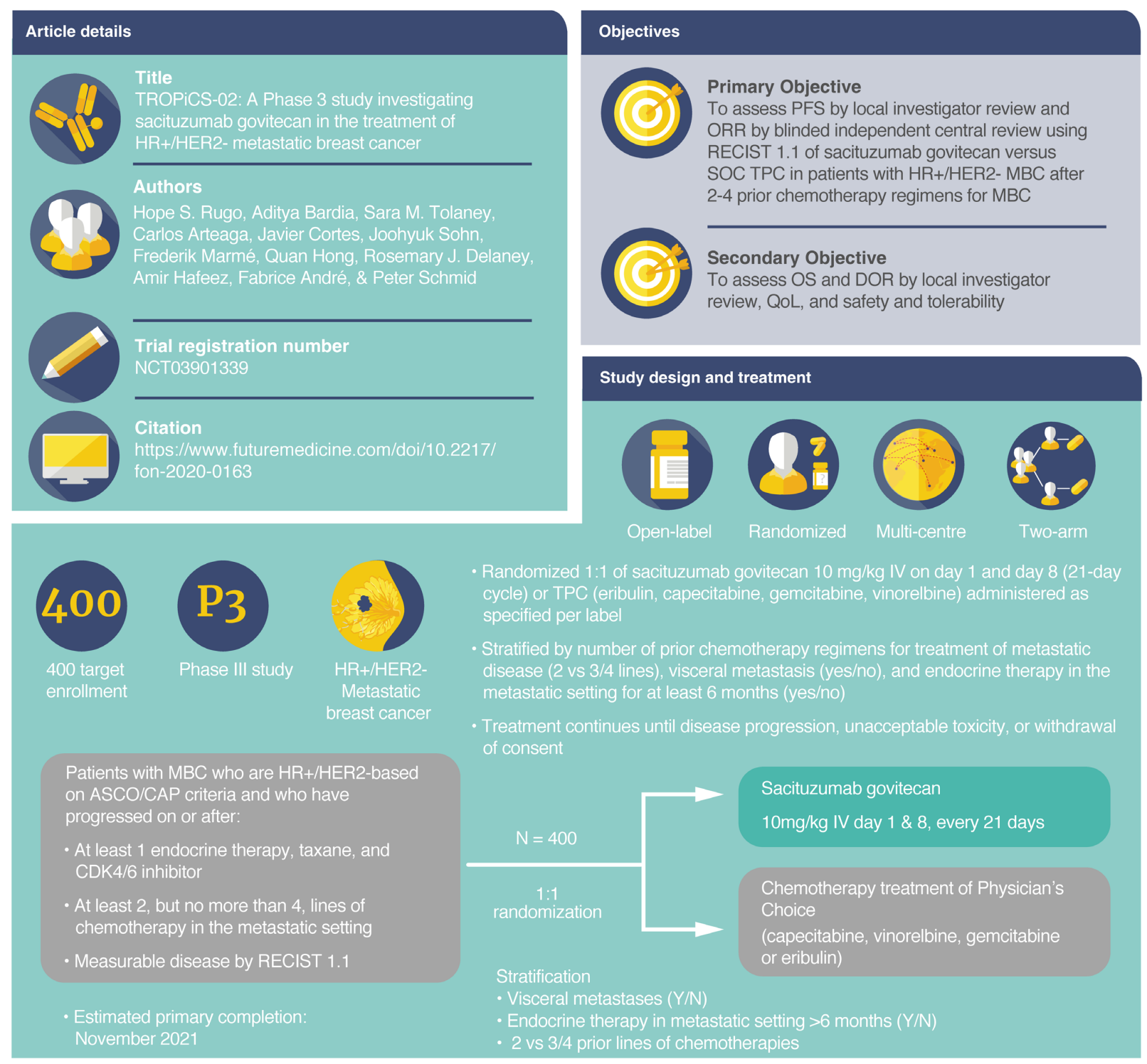

\section{Key eligibility criteria}

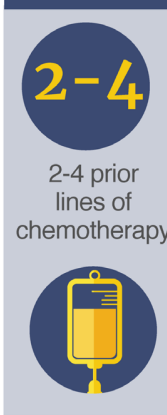

\section{- Age $>18$ years of age}

- Confirmation of HR+/HER2- MBC defined according to ASCO/CAP criteria

- Disease progression on the most recent therapy documented by CT/MRI and at least 1 measurable target lesion according to RECIST 1.1 (bone disease only is not allowed)

lines of (

Study design and treatment

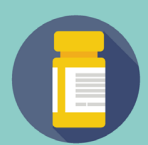

Open-label

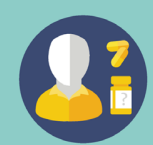

Randomized
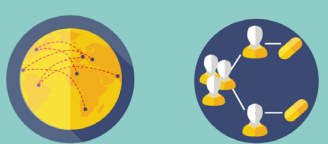

Two-arm Stratified by number of prior chemotherapy regimens for treatment of metastatic disease (2 vs 3/4 lines), visceral metastasis (yes/no), and endocrine therapy in the entatic setting for at least 6 months (yes/no)

- Treatment continues until disease progression, unacceptable toxicity, or withdrawal of consent

- 2 vs $3 / 4$ prior lines of chemotherapies

Outcome measures/endpoints

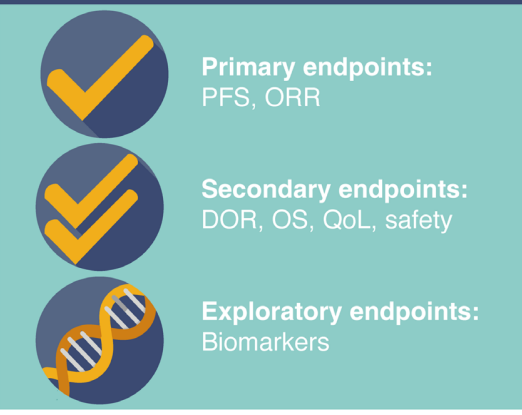

Glossary

ASCO/CAP: American Society of Clinical Oncology/College of American Pathologists; CDK: Cyclin dependent kinase; DOR: Duration of response;
CT: Computerized tomography; HER2-: Human epidermal growth factor receptor 2-negative; HR+: Hormone-receptor-positive; IV: intravenous; MBC: Metastatic breast cancer; MRI: Magnetic resonance imaging; ORR: Overall response rate; OS: Overall survival; PFS: Progression-free survival; PFS2: PFS on subsequent therapy; QoL: Quality of life; RECIST: Response Evaluation Criteria in Solid Tumors; SOC: Standard of care; TPC: Treatment of physician's choice. prior CDK $4 / 6$ inhibitor in any setting

- Eligible for one of the chemotherapy options: capecitabine, vinorelbine, gemcitabine, and eribulin 